\title{
Economic Burden of Cancer and Their Variations along with Incident Trends, Challenges, and Opportunities in India
}

\author{
Jareena Shaik ${ }^{1}$ \\ ${ }^{1}$ Vijay Institute of Medical Sciences
}

October 29, 2020

\begin{abstract}
Cancer is emerging as a major public health concern in India with the ongoing demographic and epidemiological transition. This paper uses a nationally representative household survey to look at the general prevalence and economic burden of cancer in India. The average out of pocket spending on inpatient care in private facilities is about three-times that of public facilities. These efforts should specialize in the ten cancers contributing the very best DALYs in India, including cancers of the stomach, lung, pharynx aside from nasopharynx, colon and rectum, leukemia, oesophageal, and brain, and Systema nervosum, additionally to breast, lip and oral cavity, and cervical cancer, which are currently the main target of screening and early detection programs. India's current burden of 10,00,000 incident cancers is that the results of an epidemiologic transition, improved cancer diagnostics, and improved cancer data capture. The increasing incidence of cancer in India with wide interstate variations offers useful insights and important lessons for developing countries in managing their increasing cancer burdens. Overall, the cancer epidemiology literature from India is thinly dispersed. More studies with robust designs representing all parts of the country are currently needed.
\end{abstract}

\section{INTRODUCTION}

The term "Cancer" is derived from the Greek word "Karkinos" (for crab) which refers to a generic noncommunicable disease (NCD) characterized by malignant (cancerous or neo-plasms) abnormal cells (tumor/lump) growth in any part of the human body.

However several forms of cancer have been detected, the most common sites of these tumors in human bodies are lungs, stomach, colorectal, liver, and breasts[1].

One of the major reasons for not being able to implement a screening program in India has been lack of workforce - physicians, health workers, technical staff, and pathologist to review pathological material. in increasing public awareness, supporting screening, early detection, patient and family support services, and palliative care by providing home care in these several nongovernment organizations are enagaged[2]

After cardiac diseases, as an important cause for morbidity and mortality in India cancer has emerged. The major source of information on cancer incidence and pattern in the country is The National Cancer Registry Programme and was commenced by the ICMR in December 1981.

Cancers are caused by mutations that may be inherited, induced by environmental factors, or maybe result from DNA replication errors.[5]

\section{EPIDEMIOLOGY}

Cancer is ranked as the first or second leading cause of death in 91 of 172 countries and it is third or fourth in an additional 22 countries. Cancer is that the second and fourth leading explanation for adult death in urban and rural India, respectively. 
Borrowing, sales of assets, and contributions from friends and relatives are the means through which Approximately $40 \%$ of cancer costs are met. These costs exceed $20 \%$ of annual per capita household expenditure in $60 \%$ of Indian households for the patient suffering from cancer.

Census shows that Indian citizens spent 6.74 billion US dollars in 2012 as a result of cancer deaths. from 1990 to 2016 Cancer mortality in India has doubled. India's cancer incidence is estimated to be 1.15 million for new patients in 2018 and is predicted to get almost double as a result of demographic changes alone by 2040.[5]

The low- and middle-income countries (LMICs) are undergoing an epidemiological transition, wherein the burden of communicable diseases is declining and non-communicable diseases like cancers are on the increase.

An estimation of 20 million cancer cases is expected in LMICs by 2025. There has been a considerable variation in the incidence of cancers between high-income countries (HICs) and LMICs. The incidence varies from 95/10 000 in the LMICs to over 571/100 000 in the HIC countries in both men and women.[6]

\section{METHODOLOGY}

In study 1 the survey was conducted in 2014 by the National Sample Survey Organization (NSSO), Ministry of Statistics and Program Implementation, Government of India. With a representative sample of households and randomly selected through a stratified multi-stage survey design which is covering India has conducted Social Consumption: Health Survey interviews. A rural/urban stratification is made within clusters called state-regions, which comprises an endless group of districts within a State or Union territory having similar characteristics.

This cross-sectional survey data were collected from January to June 2014. The 71st round of Morbidity and Healthcare Survey covers a sample of 65,932 households and 335,499 individuals and reported levels of cancer prevalence as well as treatment expenditure across socioeconomic categories.

The socioeconomic gradient in cancer prevalence and its healthcare utilization attentively on public and private hospitals separately concentration index (CI) is performed.[1]

In study 2 the accessible data is used from multiple sources, including 42 population-based cancer registries. The nationwide Sample Registration System of India is used to estimate the incidence of 28 types of cancer in every state of India from 1990 to 2016.

Therefore the deaths and disability-adjusted life-years (DALYs) caused by them, as a part of GBD 2016. In these death rates for all cancers together and the trends of all types of cancers, highlighting the heterogeneity in the burden of specific types of cancers across the states of India. It also presents the contribution of major risk factors to cancer DALYs in India.[3]

\section{RESULTS}

Table 1: Cancer prevalence per 100,000 persons by background characteristics and place of residence, India, National Sample Survey, 2014.

\begin{tabular}{lllllll}
\hline Background characteristics & All India & All India & Rural India & Rural India & Urban India & Urban India \\
\hline Age & Prevalence & $95 \%$ CI & Prevalence & $95 \%$ CI & Prevalence & $95 \%$ CI \\
0-14 years & & & & & & \\
15-49 years & 16 & {$[7.9,23.5]$} & 14 & {$[4.7,24.1]$} & 19 & $19[5.7,33.2]$ \\
50-59 years & 62 & {$[50.7,73.9]$} & 60 & {$[45.1,75.7]$} & 66 & {$[48.5,84.4]$} \\
60-69 years & 192 & {$[142.5,240.9]$} & 158 & {$[98.2,217.2]$} & 268 & {$[180.1,355.9]$} \\
70+ years & 321 & {$[237.5,404.6]$} & 289 & {$[182.7,394.8]$} & 391 & {$[252.6,530.4]$} \\
Sex & 385 & {$[268.4,502.3]$} & 231 & {$[107.2,355.4]$} & 727 & {$[492.3,962.7]$} \\
Male & 71 & {$[58.4,83.7]$} & 56 & {$[40.9,70.6]$} & 106 & {$[82.8,130.0]$}
\end{tabular}




\begin{tabular}{lllllll}
\hline Background characteristics & All India & All India & Rural India & Rural India & Urban India & Urban India \\
\hline Female & 96 & {$[80.7,110.5]$} & 88 & {$[68.6,106.4]$} & 115 & {$[89.9,139.7]$} \\
$\begin{array}{l}\text { Reproductive Age and Sex } \\
\text { Male: 15 to 49 years }\end{array}$ & 29 & {$[18.2,40.7]$} & 24 & {$[10.5,38.1]$} & 40 & {$[20.6,59.9]$} \\
Female: 15 to 49 years & 96 & {$[76.2,117.1]$} & 97 & {$[69.9,124.9]$} & 95 & {$[64.5,125.5]$} \\
Education & & & & & & \\
Illiterate & 79 & {$[61.7,96.0]$} & 75 & {$[54.8,96.1]$} & 93 & {$[60.8,124.4]$} \\
Primary & 53 & {$[38.6,68.1]$} & 47 & {$[29.4,64.8]$} & 71 & {$[43.1,97.9]$} \\
Secondary & 48 & {$[33.2,63.4]$} & 38 & {$[20.3,56.7]$} & 38 & {$[41.6,95.1]$} \\
Higher & 72 & {$[49.7,94.7]$} & 36 & {$[9.6,63.6]$} & 102 & {$[69.1,135.7]$} \\
MPCE quintile & & & & & & \\
Lowest & 49 & {$[33.0,65.3]$} & 33 & {$[15.0,51.0]$} & 92 & {$[59.1,124.4]$} \\
Second & 51 & {$[34.0,68.1]$} & 50 & {$[19.5,61.2]$} & 75 & {$[45.1,105.5]$} \\
Third & 61 & {$[42.1,80.4]$} & 37 & {$[16.4,57.1]$} & 112 & {$[73.9,149.8]$} \\
Fourth & 110 & {$[85.6,135.1]$} & 107 & {$[76.3,137.9]$} & 119 & {$[76.6,160.3]$} \\
Highest & 147 & {$[116.8,176.7]$} & 143 & {$[104.9,180.2]$} & 156 & {$[105.7,205.5]$} \\
Social group & & & & & \\
Scheduled tribe & 42 & {$[22.5,60.9]$} & 27 & {$[9.4,45.2]$} & 158 & {$[9.4,45.2]$} \\
Scheduled caste & 81 & {$[57.1,104.2]$} & 75 & {$[46.8,103.9]$} & 99 & {$[56.1,142.6]$} \\
Other backward classes & 89 & {$[73.1,105.1]$} & 82 & {$[61.5,101.9]$} & 107 & {$[80.1,1333.3]$} \\
Others & 89 & {$[70.4,107.1]$} & 69 & {$[45.4,93.7]$} & 114 & {$[86.3,142.4]$} \\
All India & 83 & {$[73.2,92.7]$} & 71 & {$[59.3,83.2]$} & 110 & {$[93.3,127.6]$} \\
Age-Standardized Prevalence & 97 & {$[53.2,146.1]$} & 83 & {$[28.0,141.9]$} & 130 & {$[46.0,211.4]$} \\
\hline
\end{tabular}

Table 2. Average OOP hospitalization expenditure per cancer patient by background characteristics and public and private sector treatment, India National Sample Survey 2014.

\begin{tabular}{llll}
\hline Background characteristics & Average Hospitalization Expenditure & Average Hospitalization Expenditure & Average Hospi \\
\hline & $\begin{array}{l}\text { Public sector } \\
\text { Medical }\end{array}$ & $\begin{array}{l}\text { Public sector } \\
\text { Total }\end{array}$ & Medical \\
Age & & & 55136 \\
$0-5$ years & 19805 & 30041 & 56102 \\
$6-14$ years & 32391 & 36577 & 97068 \\
$15-24$ years & 18083 & 20947 & 85441 \\
$25-59$ years & 31084 & 36665 & 65060 \\
$60+$ years & 16758 & 19912 & 101194 \\
Sex & & & 64562 \\
Male & 22782 & 27427 & 51754 \\
Female & 26448 & 30835 & 88644 \\
Education & & & 37718 \\
Illiterate & 17641 & 23176 & 121714 \\
Primary & 20495 & 24760 & \\
Secondary & 20057 & 23413 & 44500 \\
Higher & 37331 & 42232 & 44948 \\
MPCE quintile & & & 83933 \\
Lowest & & 27308 & 89809 \\
Second & 22064 & 24226 &
\end{tabular}




\begin{tabular}{llll}
\hline Background characteristics & Average Hospitalization Expenditure & Average Hospitalization Expenditure & Average Hospi \\
\hline Social group & & & 103079 \\
Scheduled tribe & 8596 & 10941 & 48389 \\
Scheduled caste & 24306 & 27977 & 74766 \\
Other backward classes & 23710 & 29528 & 94923 \\
Others & 29994 & 34015 & 72654 \\
Place of residence & & & 86941 \\
Rural & 26897 & 32202 & 78045 \\
Urban & 20686 & 24044 & \\
All India & 24523 & 29066 & \\
\hline
\end{tabular}

Table 3 Percentage of total cancer DALYs due to different types of cancers by sex in India, 2016

\begin{tabular}{llll}
\hline Both sexes combined & Both sexes combined & Females & Females \\
\hline Types of cancers & $\%$ of total cancer DALYs & Types of cancers & $\%$ of tota \\
Stomach cancer & $9 \cdot 0 \%$ & Breast cancer & $16 \cdot 8 \%$ \\
Breast cancer & $8 \cdot 2 \%$ & Cervical cancer & $10 \cdot 8 \%$ \\
Lung cancer & $7 \cdot 5 \%$ & Stomach cancer & $9 \cdot 0 \%$ \\
Lip and oral cavity cancer & $7 \cdot 2 \%$ & Colon and rectum cancer & $6 \cdot 1 \%$ \\
Pharynx cancer other than nasopharynx & $6 \cdot 8 \%$ & Lip and oral cavity cancer & $4 \cdot 6 \%$ \\
Colon and rectum cancer & $5 \cdot 8 \%$ & Ovarian cancer & $4 \cdot 6 \%$ \\
Leukemia & $5 \cdot 2 \%$ & Lung cancer & $4 \cdot 4 \%$ \\
Cervical cancer & $5 \cdot 2 \%$ & Leukemia & $4 \cdot 3 \%$ \\
Oesophageal cancer & $4 \cdot 3 \%$ & Gallbladder and biliary tract cancer & $4 \cdot 3 \%$ \\
Brain and nervous system cancer & $3 \cdot 5 \%$ & Pharynx cancer other than nasopharynx & $4 \cdot 3 \%$ \\
Liver cancer & $3 \cdot 5 \%$ & Oesophageal cancer & $3 \cdot 5 \%$ \\
Non-Hodgkin lymphoma & $3 \cdot 2 \%$ & Brain and nervous system cancer & $2 \cdot 9 \%$ \\
Gallbladder and biliary tract cancer & $3 \cdot 1 \%$ & Non-Hodgkin lymphoma & $2 \cdot 6 \%$ \\
Larynx cancer & $3 \cdot 0 \%$ & Liver cancer & $2 \cdot 3 \%$ \\
Pancreatic cancer & $2 \cdot 4 \%$ & Pancreatic cancer & $2 \cdot 2 \%$ \\
Ovarian cancer & $2 \cdot 2 \%$ & Uterine cancer & $1 \cdot 7 \%$ \\
Prostate cancer & $1 \cdot 5 \%$ & Thyroid cancer & $1 \cdot 3 \%$ \\
Bladder cancer & $1 \cdot 0 \%$ & Larynx cancer & $1 \cdot 1 \%$ \\
Nasopharynx cancer & $1 \cdot 0 \%$ & Multiple myeloma & $1 \cdot 0 \%$ \\
Multiple myeloma & $1 \cdot 0 \%$ & Nasopharynx cancer & $0 \cdot 7 \%$ \\
Hodgkin's lymphoma & $0 \cdot 9 \%$ & Hodgkin's lymphoma & $0 \cdot 6 \%$ \\
Uterine cancer & $0 \cdot 8 \%$ & Bladder cancer & $0 \cdot 6 \%$ \\
Kidney cancer & $0 \cdot 7 \%$ & Kidney cancer & $0 \cdot 4 \%$ \\
Mesothelioma & $0 \cdot 3 \%$ & Malignant skin melanoma & $0 \cdot 2 \%$ \\
Malignant skin melanoma & $0 \cdot 3 \%$ & Mesothelioma & $0 \cdot 2 \%$ \\
Testicular cancer & $0 \cdot 2 \%$ & Non-melanoma skin cancer & $0 \cdot 1 \%$ \\
Non-melanoma skin cancer & $0 \cdot 2 \%$ & & \\
\hline
\end{tabular}

\section{INDICATORS OF SOCIOECONOMIC STATUS (SES)}

Hose hold monthly per capita expenditure (MPCE) quintile, education, and social group of the cancer patient are the three SES indicators on which mainly focused. Indian education system categorized the patients as illiterate (no formal schooling), primary education or below (1-5 years), middle school or below (6-10years), secondary education (11-12 years ) and higher education (graduate school and above). Social groups were 
divided as scheduled tribes (ST), scheduled castes (SC), other backward classes, and other castes. SC and ST groups were economically, socially, and geographically deprived groups in India when compared to other castes which are having better SES.[1]

\section{RISK FACTORS}

In the Indian subcontinent, the most common risk factor studied was tobacco in gastrointestinal cancers, head and neck cancers, lung cancers, prostate cancer, and urinary tract cancers. $[7,8,9]$

The estimated risk in male and female cancer patients is $45 \%$ and $17 \%$ respectively. Papillomavirus in oral cancer is a good example of cultural practices affecting risk factors.[10]

Female is commonly affected by cervical cancer in India and it is the second most common form.[11]

Studies on Tobacco-related cancers are highest in numbers, proportionate to their prevalence but cervix and breast cancers need to be explored for their risk factors as the number of studies for modifiable risk factors for these two cancers are low. The design of the risk factor studies reviewed if lacks any randomized control trials (RCT) and large prospective cohorts. It may be due to the complexity of conducting RCT and high costs. Most of the studies reviewing risk factors have incorporated cross-sectional or case-control study designs for their studies.[12]

\section{INCIDENCE AND TRENDS}

It is not clear whether withholding or no disclosure of the funding source was the result of either the lack of significant funding or potential conflict of interest. We have included manuscripts published in PubMed only, and other databases are excluded. It also missed some of the data published as government reports and gazettes.[6]

Incidence and trends are studied by Seventy-six papers. uniformity was not found in reporting the incidence rates. The incidence has been reported from the population-based registries and trends from secondary data as ASR and expected annual percentage change. The percentage of patients treated represents the incidence of hospital-based studies. The non-uniformity in reporting and predominance of hospital-based registries lead to misrepresentation of the incidence of cancers and Keeping Journal of Medicine had also revised its rules about conflict of interest.[13 ]

\section{STRATEGIES FOR EARLY DETECTION OF COMMON CANCERS IN INDIA}

The cancers of the oral cavity, uterine cervix, and female breast are very susceptible to early detection. Periodic examination for early detection of cervix and breast cancers in the developed countries, Pap smear, and Mammography are the accepted standards. in India Pap smear and mammography are however not practical and affordable methods for cervix and breast cancer screening.[14,15]

\section{MODEL CANCER CONTROL PROGRAM}

A comprehensive cancer control program in the backward Ratnagiri and Sindhudurg districts of Maharashtra has been started by The Tata Memorial Hospital. This program proposes to cover the eligible population of these districts with two rounds of screening for oral, cervix and breast cancers at two-year intervals.

Trained primary health care workers will perform screening. The treatment of the screened positive cases will be carried out locally at an NGO Hospital at Chiplun in Ratnagiri, the BKL Walavalkar Hospital. This program, which was started in August 2003, is an 'Xth-plan Project' of the Department of Atomic Energy that will be completed by March 2007 and is expected to form a model for district cancer control programs in the country.[4]

\section{THE FUTURE OF CANCER IN INDIA}

India's epidemiologic transition was triggered by large reductions in premature deaths from infections and associated diseases and increased life expectancy. an increase in cancer and other non-communicable diseases are experienced by all Indian states.[16] Due to a lack of adequate and easily accessible cancer care facilities, 
in the least developed and rural parts of India, Cancer diagnoses are still missed. In 1993, an autopsy study from India's premier postgraduate medical institute revealed that $25.8 \%$ of cancers were incorrectly diagnosed.[17]

There are still chances of error in Indian urban cancer registries in terms of data quality.[18] The 2018 quality report from Cancer Incidence in Five Continents indicates that $23 \%$ of cancers in rural Assam were unclassifiable.[19] The increasing availability of minimally invasive diagnostic technologies, including image-guided needle aspiration cytology and immunohistochemistry, will further increase cancer diagnosis in India.[20] The introduction of computed tomography scanning in Mumbai in the mid-1980s was immediately followed by an increase in the incidence of brain tumors, which stabilized later.[21]

The reduction of cardiovascular disease mortality is correlated with increased cancer mortality in many developed countries. [22] Further reduction in cardiovascular disease mortality, which is presently three times higher than cancer in India, will increase the cancer burden further. Cancer screening, which is being considered by the GOI, is known to increase incidence while reducing mortality.[23]

\section{DISCUSSION AND CONCLUSION}

Increasing the prevalence of cancer is a major public health concern. the overall self-reported prevalence of cancer is estimated to be 83 per 100,000 persons with a greater prevalence in urban areas (110 per 100,000 persons).[10] In addition to this, cancer prevalence for the age-standardized rate is estimated to be 97 per 100,000 persons. These estimates are also similar to the age-adjusted cancer incidence (94 per 100,000 persons) discussed.[24]

According to table 1, cancer prevalence is highest in urban India when compared to rural India concerning its background characteristics

According to Table 2, the average hospitalization expenditure is greater for the private sector when compared to the public sector following its background characteristics

According to Table 3, the highest percentage of total cancer DALYs is stomach cancer and the lowest percentage of total cancer DALYs is testicular cancer and non-melanoma skin cancer in both sexes combined

In females, the highest percentage of total cancer DALYs is breast cancer, and the lowest percentage of total cancer DALYs is non-melanoma skin cancer. In males, the highest percentage of total cancer DALYs is breast cancer and the lowest percentage of total cancer DALYs is non-melanoma skin cancer, breast cancer, and malignant skin melanoma

The higher burden of cancer among the elderly cohort and in demographically advanced states implies greater requirements of tertiary care facilities. [25]

Cervical cancer is ranked as most frequently caused by cancer among women which are mainly caused by sexually transmitted human papillomavirus (HPV). About three fourth of sexually active adults are likely to be affected by any one type of HPV which is suggested in many studies.[26]

The Indian Academy of Pediatrics and Committee on immunization (IAPCOI) recommends offering vaccines to only those who can afford but HPV vaccination is of public health concern. Policies should be made to check universal risk factors causing cancerous tumors such as tobacco and alcohol, poor diet (insufficient fruit or vegetable intake), overweight and obesity, physical inactivity, chronic infections from Hepatitis B and $\mathrm{C}$ virus, and environmental risks including ionizing and non-ionizing radiation. [27]

Between 1992 and 2012, India has a third-highest increase in alcohol per capita (APC) among 40 countries.[28]

After the USA and China, India has the third-highest number of obese individuals in the world. If detected at the right stage properly, half of the cancer cases can be successfully treated which is suggested in a few studies. [29] 
In India, the detection rate is very low and about only 20 to 30 percent of cases are diagnosed at Stage I and II respectively which indicates the increase in general awareness regarding cancer symptoms, causes, prevention, and measure and treatment options. Although, in 1984 with four major goals i.e. primary prevention of tobacco-related cancers, early detection of cancers, augmentation of treatment facilities, and establishing palliative care is formulated by the National Cancer Control Programme (NCCP) was formulated.[30]

National Cancer Registry Programme (1982) has been providing authentic information on cancer incidence since more than 30 years, but the functioning of NCRP is according to just 28 Population-Based Cancer Registries (PBCRs)

It is important to reiterate that cancer treatment in India should be received as a priority both to improve cancer survival and to protect households from financial catastrophe. As this study mainly aims at analyzing out of pocket expenditure and financial hardships on cancer inpatient treatment, information on availability and cost of drugs, access to modern techniques of treatment is also desirable, estimates on catastrophic expenditure at different thresholds (i.e. 10\%, 20\%, and 30\%) across different population groups does-not reveal the information about the willingness of households to spend on cancer care. [31]

\section{ACKNOWLEDGMENT}

We acknowledge the Vijaya Institute of Pharmaceutical Sciences For Women for support and valuable guidance.

\section{CONFLICT OF INTEREST}

We declare that we have no conflict of interest.

\section{REFERENCES}

1. Sunil Rajpal*, Abhishek Kumar, William Joe, Economic burden of cancer in India: Evidence from the cross-sectional nationally representative household survey, 2014

2. Cancer research in India: Challenges \& opportunities, Indian J Med Res 148, October 2018, pp 362-365

3. India State-Level Disease Burden Initiative Cancer Collaborators, The burden of cancers and their variations across the states of India: the Global Burden of Disease Study 1990-2016, the lancet oncology, volume 19, issue10, P1289-1306, October 01, 2018

4. *Dinshaw KA, Shastri SS, Patil SS, CANCER CONTROL PROGRAMME IN INDIA: CHALLENGES FOR THE NEW MILLENNIUM, Health Administrator Vol: XVII, Number 1: 10-13, pg

5. Robert D Smith and Mohandas K. Mallath, MD, DNB. History of the Growing Burden of Cancer in India: From Antiquity to the 21st Century. J Glob Oncol. 2019 Jul;5:1-15.

6. K.V. Deepa, Jubina Balan Venghateri, Monty Khajanchi, Anita Gadgil, Nobhojit Roy, Cancer epidemiology literature from India: Does it reflect the reality?, Journal of Public Health, 27 December 2019, pii: fdz160.

7. Ramadas K, Sauvaget C, Thomas G et al. Effect of tobacco chewing, tobacco smoking, and alcohol on all-cause and cancer mortality: a cohort study from Trivandrum, India. Cancer Epidemiol 2010;34:40512

8. ICMR NCRP. A consolidated report of hospital-based cancer registries [Internet]. 2012;

9. Sinha DN, Abdulkader RS, Gupta PC. Smokeless tobacco-associated cancers: a systematic review and meta-analysis of Indian studies. Int J Cancer 2016; Mar 15; 138(6): 1368-79 DOI: 10.1002/ijc.29884

10. Laprise C, Madathil SA, Allison P, et al. No role for human papillomavirus infection in oral cancers in a region in southern India. Int $\mathrm{J}$ Cancer 2016;138:912-7

11. WHO. NCD Country Profile. Geneva: World Health organization; 2011.doi: http://www.who.int/nmh/ countries/ind_en.pdf?ua=1

12. Davidoff F, DeAngelis CD, Drazen JM et al. Sponsorship, authorship, and accountability. JAMA 2001;286:1232-4

13. Gottlieb S. New England journal loosens its rules on conflict of interest. BMJ 2002;324:1474

14. IARC Working Group on Cervical Cancer Screening. Summary Chapter. In: Hakama M, Miller AB, 
Day NE, (eds). Screening for Cancer of the Uterine Cervix. IARC Scientific Publications No. 76. Lyon, International Agency for Research on Cancer, 1986.

15. Sankaranarayanan R Budukh A, Rajkumar R. Effective screening programs for cervical cancer in lowand middle-income developing countries

16. Indian Council of Medical Research, Public Health Foundation of India, Institute for Health Metrics and Evaluation: GBD India Compare Data Visualization: New Delhi-ICMR, PHFI, and IHME, 2017. http://vizhub.healthdata.org/gbd-compare/india

17. Sarode VR, Datta BN, Banerjee AK, et al: Autopsy findings and clinical diagnoses: A review of 1,000 cases. Hum Pathol 24:194-198, 1993

18. .Mathew A, Daniel CR, Ferrucci LM, et al: Assessment of follow-up, and the completeness and accuracy of cancer case ascertainment in three areas of India. Cancer Epidemiol 35:334-341, 2011.

19. International Agency for Research on Cancer: Indices of data quality http://ci5.iarc.fr/CI5XI/PDF/INDICES/unspecified.pdf

20. .Sehgal IS, Dhooria S, Aggarwal AN, et al: Impact of rapid on-site cytological evaluation (ROSE) on the diagnostic yield of transbronchial needle aspiration during mediastinal lymph node sampling: Systematic review and meta-analysis. Chest 153:929-938, 2018

21. Yeole BB: Trends in brain cancer incidence in India. Asian Pac J Cancer Prev 9:267-270, 2008

22. Weir HK, Anderson RN, Coleman King SM, et al: Heart disease and cancer deaths: Trends and projections in the United States, 1969-2020. Prev Chronic Dis 13:E157, 2016

23. Sivaram S, Majumdar G, Perin D, et al: Population-based cancer screening programmes in low-income and middle-income countries: Regional consultation of the International Cancer Screening Network in India. Lancet Oncol 19:e113-e122, 2018

24. Liff JM, Sung JF, Chow WH, et al: Does increased detection account for the rising incidence of breast cancer? Am J Public Health 81:462-465, 1991

25. Mallath MK, Taylor DG, Badwe RA, Rath GK, Shanta V, Pramesh CS, et al. The growing burden of cancer in India: epidemiology and social context. The Lancet Oncology. 2014; 15: DOI e205-e212.

26. National Cancer Registry Program. Incidence, Distribution, rends in incidence rates, and projections of burden of cancer. Indian Council of Medical Research; 2016.

27. Kaarthigeyan K. Cervical cancer in and HPV vaccination. International journal of India Medical and Pediatric Oncology. 2012; 33: 7-11.

28. Rock CL, Lampe JW, Patterson RE. Nutrition, genetics, and risk of cancer. Annual Review Public Health. 2000; 21: 47-64.

29. Kaarthigeyan K. Cervical cancer in and HPV vaccination. International journal of India Medical and Pediatric Oncology. 2012; 33: 7-11.

30. Li CI, Rossing MA, Voigt LF, Daling JR. Multiple primary breasts and thyroid cancers: Role of age at diagnosis and cancer treatments (United States). Cancer Causes and Control. 2000; 11: 805-811. PMID: 11075869

31. Ramadas K, Arrosi S, Thara S, Thomas G, Jissa V, Fayette JM, et al. Which socioeconomic factors are associated with participation in oral cancer screening in the developing world? Results from a population-based screening project in India. Cancer Detection and Prevention. 2008; 32: 109-115. https://doi.org/10.1016/j.cdp.2008.02.008 PMID: 18632218

32. Dinshaw K A, Shastri SS, Patil SS. cancer control program in India: Challenges for the new millennium. Health Administrator. 2010; 17: 10-13. 